\title{
Stability Analysis of the LHC Cables for Transient Heat Depositions
}

\author{
Pier Paolo Granieri, M. Calvi, P. Xydi, B. Baudouy, D. Bocian, L. Bottura, M. Breschi, and A. Siemko
}

\begin{abstract}
The commissioning and the exploitation of the LHC require a good knowledge of the stability margins of the superconducting magnets with respect to beam induced heat depositions. Previous studies showed that simple numerical models are suitable to carry out stability calculations of multi-strands cables, and highlighted the relevance of the heat transfer model with the surrounding helium. In this paper we present a systematic scan of the stability margin of all types of LHC cables working at $1.9 \mathrm{~K}$ against transient heat depositions. We specifically discuss the dependence of the stability margin on the parameters of the model, which provide an estimate of the uncertainty of the values quoted. The stability margin calculations have been performed using a zero-dimensional (0-D) numerical model, and a cooling model taking into account the relevant helium phases which may appear during a stability experiment: it includes Kapitza thermal resistance in superfluid He, boundary layer formation and heat transfer in He I, and considers the transition from nucleating boiling to film boiling during He gas formation.
\end{abstract}

Index Terms-Heat transfer coefficients, helium phases, LHC superconducting cables, stability margins, transient heat depositions.

\section{INTRODUCTION}

$\mathbf{O}$ NE of the issues for the operation of the Large Hadron Collider (LHC) [1] are the quenches induced in the superconducting (SC) magnets by lost particles from high energy and high intensity proton beams. In order to cope with this problem, a control system has been developed to predict an imminent beam induced quench and dump the beam before quenching the magnet. This system is based on detectors, the beam loss monitors (BLM), which measure the flux of secondary particles (hadronic shower) produced during the collision of protons with the beam screen, the cold bore and the superconducting coils [2]. Reaction thresholds for the BLM's need to be set comparing the energy deposition due to the hadronic shower (computed with specific codes [3]) to the expected stability of the SC cables in the coil. The efficiency of the BLM system will obviously depend on the precision of the estimation of the stability margin of the SC cables, which is the aim of this work. Similar studies have been carried out by various authors [4]-[6], without investigating the response of the system to different perturbation

Manuscript received August 28, 2001

P. P. Granieri is with CERN, Geneva, Switzerland. He is also with Bologna University, Italy (e-mail: Pier.Paolo.Granieri@cern.ch).

M. Calvi is with EPFL-CRPP, Switzerland.

P. Xydi, L. Bottura, and A. Siemko are with CERN, Geneva, Switzerland.

B. Baudouy is with CEA-Saclay DSM/DAPNIA/SACM, France.

D. Bocian is with CERN, Geneva, Switzerland. He is also with IFJ PAN Krakow, Poland.

M. Breschi is with Bologna University, Italy.

Digital Object Identifier 10.1109/TASC.2008.922543 times, and with a cooling model only constituted by the He II and film boiling phases.

The work presented here is the follow-up of a previous investigation which set the methodology to face such problems [7], simulating numerically a resistive transition in a multi-strands SC cable and evaluating iteratively the stability against external heat depositions. This allowed determining that the most relevant parameter for stability calculations is the heat transfer coefficient between strands and $\mathrm{He}$, and that a simple easy-to-handle 0-D model can be used instead of more complex ones. Indeed it gives a good estimate of the stability for energy depositions over a sufficiently long length, in particular above $10 \mathrm{~cm}$, which is the case when considering transient distributed disturbances such as beam loss. This first study only focused on the inner layer cable of the LHC dipoles at the nominal operating current and in the peak field region.

In this paper, particular attention is paid to build a detailed model describing the transient heat exchange between strands and $\mathrm{He}$, depending on different $\mathrm{He}$ phases. The stability of all the LHC SC cables working at an operational temperature of $1.9 \mathrm{~K}$ is numerically computed with respect to the actual range of beam loss perturbation times. A wide current range is covered, also considering cables in various field regions. Furthermore, the impact of the different He phases on results is discussed, and a parametric study on the effectiveness of the $\mathrm{He}$ micro-channels outside the cables and of the He channels network through the cable insulation is shown.

\section{MODEL DESCRIPTION}

As already mentioned, a 0-D model can be used thus neglecting the longitudinal cable dimension (and consequently heat conduction in this direction), the details of the He flow and the current distribution phenomena. Moreover, the strands in the cable cross-section are lumped into a single thermal component characterized by uniform temperature and homogenized thermal properties. A companion paper [8] demonstrated the consistency of this approach which is appropriate when considering heating sources distributed across the whole cable cross-section.

The goal of the present work is to obtain the stability margin defined as the maximum energy per unit volume of cable that can be tolerated still leading to a recovery in the specific operating conditions (temperature, current and field) and perturbation spectrum considered. Transient perturbations are modeled by a square heating waveform.

The thermal network in the cable cross-section is constituted by the strands, the $\mathrm{He}$ content inside the cable, the insulation wrapped around the cable and the external superfluid He bath at the constant temperature $T_{b}=1.9 \mathrm{~K}$ (see Fig. 1). The first three components are all thermally coupled, as in the original 


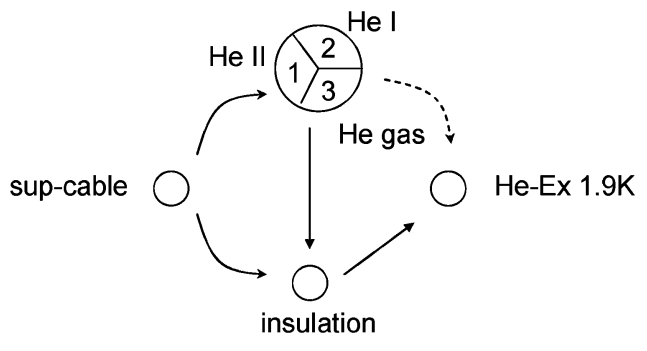

Fig. 1. The thermal network implemented in the model.

0-D model [9]. In addition, heat exchange between the cable insulation and the external bath and between the He fraction in the cable and the external bath (by He II contained inside the insulation) is allowed.

This thermal network is described by a system of ordinary differential equations based on a local balance of energy:

$$
\left\{\begin{array}{l}
A_{s} \rho_{s} C_{s} \frac{\partial T_{s}}{\partial t}=\dot{q}_{\text {ext }}^{\prime}+\dot{q}_{J o u l e}^{\prime}-p_{s, h} h_{s, h}\left(T_{s}-T_{h}\right) \\
-p_{s, i} h_{s, i}\left(T_{s}-T_{i}\right), \\
A_{h} \rho_{h} C_{h} \frac{\partial T_{h}}{\partial t}=p_{s, h} h_{s, h}\left(T_{s}-T_{h}\right)+p_{i, h} h_{i, h}\left(T_{i}-T_{h}\right) \\
-\left(p_{i, h}+p_{s, i}\right) Q_{H e I I}, \\
A_{i} \rho_{i} C_{i} \frac{\partial T_{i}}{\partial t}=-p_{i, h} h_{i, h}\left(T_{i}-T_{h}\right)-p_{s, i} h_{s, i}\left(T_{i}-T_{s}\right) \\
-p_{i, b} h_{i, b}\left(T_{i}-T_{b}\right),
\end{array}\right.
$$

where the subscripts refer to the strands $(s)$, He fraction in the cable $(h)$, insulation $(i)$ and external He bath $(b)$. The left hand side of (1) contains the heat capacity of each element, where $A$ is its cross-section, $\rho$ its density, $C$ its specific heat at constant pressure and $T$ its temperature, which is considered uniform over the component cross-section. The right hand side of (1) contains the external heat perturbation $\dot{q}_{\text {ext }}^{\prime}$, the Joule heat $\dot{q}_{J o u l e}^{\prime}$ and the heat exchanged between all the components at their mutual interface. The latter is expressed in terms of contact (or wetted) perimeters $p$ and surface heat transfer coefficients $h$. As regards the heat transfer coefficients, the Section III is devoted to the description of the $h_{s, h}$ between strands and $\mathrm{He}$ inside the cable. For the definition of the other $h$ 's, the following expressions have been used:

$$
h_{s, i}=\frac{h_{s} h_{i}}{h_{s}+h_{i}} ; \quad h_{i, h}=\frac{h_{i} h_{s, h}}{h_{i}+h_{s, h}} ; \quad h_{i, b}=\frac{h_{i} h_{b}}{h_{i}+h_{b}},
$$

where the empirical definitions $h_{i}=2 K_{i} / t_{i}$ and $h_{s}=$ $1000 \mathrm{~W} / \mathrm{m}^{2} \mathrm{~K}$ define the thermal contact of strands and insulation [9], while $1 / h_{b}$ is the Kapitza resistance between the insulation and the external bath. $K_{i}$ is the insulation thermal conductivity and $t_{i}$ is its thickness. The term $Q_{\mathrm{HeII}}$ in the (1) allows taking into account the thermal coupling between the $\mathrm{He}$ inside the cable and the external bath through the complicated He channels network between the insulation layers and through the insulation porosity. As in [10], the heat flux in Gorter-Mellink regime $Q_{\mathrm{HeII}}$ is modeled defining an equivalent channel in terms of heat transport property and assuming that He II heat transfer in the channels and conduction in the insulation are independent:

$$
Q_{H e I I}=\frac{1}{A_{t}}\left(\frac{A}{L^{1 / 3}}\right)\left[\int_{T_{b}}^{T_{h}} f(T) d T\right]^{1 / 3},
$$

where the cross-section $A$ and the length $L$ of the equivalent channel mentioned above define the geometrical group $G=$ $\left(A / L^{1 / 3}\right)$; the normalization to the heat transfer surface $A_{t}$ of the experimental apparatus in [10] is required, and $f(T)$ is the superfluid thermal conductivity.

We make the hypothesis that He inside the cable can escape the cable interstices, so that the pressure remains close to its initial value of 1.3 bar, and the density can be obtained using the state equation $\rho(p, T)$.

A more complete model should also take into account the $\mathrm{He}$ flow in longitudinal and radial direction to evaluate the increase of pressure due to the temperature increase, and should allow having supercritical instead of boiling He when the critical pressure of 2.3 bar is exceeded.

An important feature of this model is to link the transient to the steady state regime (discussed in detail in [11]), where the stability does not depend anymore on the enthalpy of the components but only on the heat transfer path between the strands and the reservoir.

\section{Heat Transfer to Helium}

The theoretical evaluation of the heat transfer coefficient between the strands and the He filling the interstices among them represents a complex task. It depends on several parameters, which are often unknown and difficult to measure.

The model adopted in this study consists of the composition of different terms related to the different He phases, as summarized below:

$$
h_{s, h}= \begin{cases}h_{K} & T_{h} \leq T_{\lambda}, \\ h_{\text {HeI }} & T_{\lambda}<T_{h}<T_{\text {Sat }}, \\ h_{\text {nucl.boil. }} . & T_{h}=T_{\text {Sat }} \rightarrow\left(T_{h}=T_{\text {Sat }}\right), \\ h_{\text {film }} & E_{\text {film }}=E_{\lim } \rightarrow\left(T_{h}=T_{\text {Sat }}\right), \\ h_{\text {gas }} & E_{\text {gas }}=E_{\text {lat }} \rightarrow\left(T_{h}>T_{\text {Sat }}\right) .\end{cases}
$$

At the beginning of the thermal transient the surface temperature of the strands $T s$ is below the lambda temperature $T_{\lambda}$, and the heat exchange with He II is limited by the Kapitza resistance at the interface between the He and the strands. The Kapitza heat transfer coefficient can be approximated by:

$$
h_{K}=\sigma\left(T_{s}^{2}+T_{h}^{2}\right)\left(T_{s}+T_{h}\right),
$$

where the constant $\sigma$ depends on the nature and surface state of the strands and is taken equal to $200 \mathrm{~W} / \mathrm{m}^{2} \cdot \mathrm{K}^{4}$.

When all the He surrounding the strands reaches the $T_{\lambda}$, the He I phase starts and temperature gradients are established in the He bulk. Under a sustained heating, the boundary between the $\mathrm{He}$ I and He II, the so-called $\lambda$-front, starts propagating while the He I phase grows. At this point, a thermal boundary layer forms at the strands-He interface. The temperature profile in the boundary layer can be approximated by a heat diffusion process in a semi-infinite body (the $\mathrm{He}$ ) due to a heat flux step at the surface. The equivalent heat transfer coefficient is given by [7]:

$$
h_{B L}=\frac{1}{2} \sqrt{\frac{K_{h} \rho_{h} c_{h}}{\pi \Delta t}},
$$

where $K_{h}$ is the thermal conductivity of He and $\Delta t=t-t_{\lambda}$, where $t_{\lambda}$ is the time at which the transition into He I starts. After the boundary layer is fully developed, the heat transfer mechanism is driven by a steady state heat transfer coefficient, which has been estimate to be $h_{s s}=50 \mathrm{~W} / \mathrm{m}^{2} \cdot \mathrm{K}$ after evaluation 
of the corresponding minimum Nu number. The transition from the transient to the steady state heat transfer in the boundary layer takes place when the temperature in the boundary layer reaches the steady state, linear profile. In our case we have approximated this transition following one of the two approaches proposed by Arp [12]:

$$
h_{H e I}=\max \left\{\frac{h_{K} h_{B L}}{h_{K}+h_{B L}} ; h_{s s}\right\}
$$

Once the saturation temperature $T_{S a t}$ is reached, the He enters the nucleate boiling phase, described by a Kapitza heat transfer coefficient $h_{\text {nucl.boil }}$ as defined in [13], [14]:

$$
h_{\text {nucl.boil. }}=\frac{\beta\left(T_{s}^{m}-T_{h}^{m}\right)}{T_{s}-T_{h}},
$$

where the parameters $\beta$ and $m$ assume the values of $242 \mathrm{~W} / \mathrm{m}^{2}$. $\mathrm{K}^{m}$ and 2.8 respectively, which allow the best fit of the experimental results in [14].

The nucleate boiling phase would last indefinitely long if the heat flux towards He does not exceed the critical heat flux, or the surface superheat does not exceed a given threshold $\Delta T^{*}$. If one of these thresholds is overcome the nucleate boiling phase cannot evacuate anymore heat without forming a film boiling [13], so energy starts accumulating into the He. This energy $\left(E_{\text {film }}\right)$ is integrated starting from the instant $t_{0}^{*}$ when $T_{s}$ equals $T_{\text {Sat }}+\Delta T^{*}$, and compared with the total energy flux $E_{\text {lim }}$ needed to form film boiling [14]:

$$
E_{\lim }=\alpha\left(t_{o n}-t_{0}^{*}\right)^{n},
$$

where $t_{o n}$ is the onset time of film boiling, $\alpha=720 \mathrm{~J} / \mathrm{m}^{2} \mathrm{~s}^{\mathrm{n}}$ and $n=0.6$. When $E_{\text {film }}$ equals $E_{l i m}$ the transition to film boiling occurs and consequently the heat transfer coefficient drops to its film boiling value $h_{\text {film }}$. It is taken equal to a constant value of $250 \mathrm{~W} / \mathrm{m}^{2} \cdot \mathrm{K}$, according to the most of the literature [4]-[6], [13]-[15]. This energetic criterion also takes into account the case of variable heat fluxes, and the very good agreement obtained with experimental results [14] confirms its reliability. This criterion, developed for a He bath, is adapted to the case of narrow channels considering the measured reduction of critical heat flux with reducing the channel width [15]. This leads to a reduction of $\Delta T^{*}$, that for our case is set to $0.4 \mathrm{~K}$.

It is relevant to notice that the film thickness increases with the strands temperature. Unlike the case of a bath, in which the film always acts as a thermal barrier between the strands and a liquid He reservoir, in narrow channels this is the case as long as the film thickness is smaller than the channel width [16]. Since the whole $\mathrm{He}$ in the channel is vaporised $\left(E_{\text {gas }}=E_{\text {lat }}\langle=\rangle\right.$ film thickness equal to the channel width), the worsening of the heat transfer observed in the quoted experimental work is taken into account allowing transition to a totally gaseous phase. This is described by a constant heat transfer coefficient $h_{\text {gas }}$ of $\sim 70 \mathrm{~W} / \mathrm{m}^{2} \cdot \mathrm{K}$, as extrapolated from experiments performed in similar geometric conditions [15], [17].

It is worth noting that during the phase transition (nucleate and film boiling) the He temperature is kept constant, whereas it can vary in the other phases. This feature, together with the

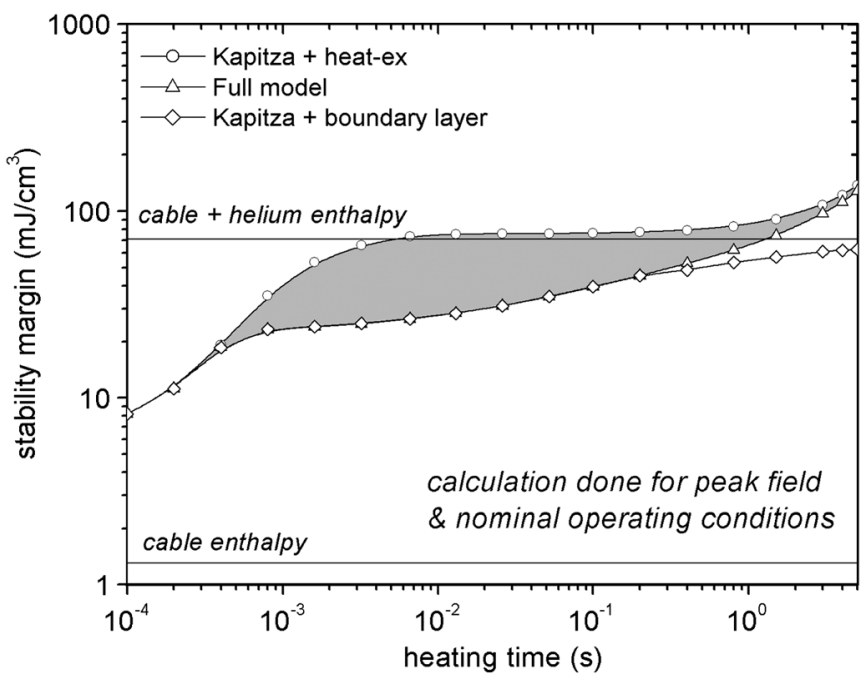

Fig. 2. Stability margin of cable 1 at nominal current and peak field as a function of heating time. Three different heat transfer models (between the strands and the He fraction in the cable) have been considered. Also the enthalpy of the cable components is reported.

definition of a $h_{\text {gas }}$ lower than $h_{\text {film }}$, contributes to decrease the heat exchange towards He during the gaseous phase.

The heat transfer model implemented in this study is irreversible, in the sense that no recovery to a previous He phase is allowed. This choice is conservative, but the impact of a more realistic reversible model is being investigated.

Details of the heat transfer model will be given in a following, dedicated publication.

\section{RESULTS}

The stability margin of the LHC cables working at $1.9 \mathrm{~K}$ have been estimated for different heating times, ranging from $100 \mu \mathrm{s}$ up to $1 \mathrm{~s}$. The field versus current transfer function used for the calculation is relative to the cable in the peak field region of the magnet cross section. It is convenient to approach the discussion of the results starting with the estimation of the impact of the $\mathrm{He}$ transfer model and of the cooling surface, to better understand the overall mechanisms underlying the stability of the system.

\section{A. Impact of the Heat Transfer Model}

Several heat transfer coefficients have been introduced which affect the system at different times of its evolution. The technique used to estimate their impact on the final results is presented in Fig. 2 where the stability margin is calculated as a function of the heating time for different heat transfer models. The inner layer cable of the main dipoles (cable 1 in Table I) is considered. The comparison with the enthalpy reserve (between $T_{b}$ and the current sharing temperature) of both the dry cable (without $\mathrm{He}$ ) and the wet cable is also shown. The results using only the Kapitza heat transfer coefficient are by far the most optimistic. This simplified and unrealistic model compared with the final model nevertheless highlights that the Kapitza regime dominates up to $400 \mu \mathrm{s}$ time scale. For longer pulses the $T_{\lambda}$ is reached and the limitations in the heat transfer due to the presence of He I (and/or gas phase) become important. This is true up to $5 \mathrm{~s}$ pulse duration, when the heat transfer through the insulation becomes effective and the two curves become again identical. At this current regime (about $50 \%$ of the critical current) 
TABLE I

Main Operating Parameters of THE LHC CABLES Working AT $1.9 \mathrm{~K}$

\begin{tabular}{|c|c|c|c|c|c|}
\hline & & $\begin{array}{c}\text { Cable } 1 \\
(\mathrm{MB})\end{array}$ & $\begin{array}{c}\text { Cable } 2 \\
(\mathrm{MB})\end{array}$ & $\begin{array}{c}\text { Cable } 3 \\
\text { (MQ) }\end{array}$ & $\begin{array}{l}\text { Cable } 4 \\
(\mathrm{MQM})\end{array}$ \\
\hline Strand diameter & $(\mathrm{mm})$ & 1.065 & 0.825 & 0.825 & 0.48 \\
\hline Number of strands & & 28 & 36 & 36 & 36 \\
\hline $\mathrm{r}=\mathrm{Cu} / \mathrm{Nb}-\mathrm{Ti}$ & & 1.65 & 1.95 & 1.95 & 1.75 \\
\hline Cross-sections: $\mathrm{Cu}$ & $\left(\mathrm{mm}^{2}\right)$ & 15.871 & 13.203 & 13.203 & 4.229 \\
\hline $\mathrm{Nb}-\mathrm{Ti}$ & $\left(\mathrm{mm}^{2}\right)$ & 9.618 & 6.771 & 6.771 & 2.417 \\
\hline $\mathrm{He}$ & $\left(\mathrm{mm}^{2}\right)$ & 2.005 & 1.494 & 1.627 & 0.429 \\
\hline Insulation (Polyimide) & $\left(\mathrm{mm}^{2}\right)$ & 5.460 & 5.327 & 4.510 & 1.885 \\
\hline Radial / Azimuthal & $(\mathrm{mm})$ & $0.15 /$ & $0.15 /$ & $0.13 /$ & $0.08 /$ \\
\hline insulation thickness & (IIIII) & 0.12 & 0.13 & 0.11 & 0.08 \\
\hline Nominal current & (A) & 11850 & 11850 & 11870 & 5390 \\
\hline $\begin{array}{l}\text { Peak field@ } \\
\text { nominal current }\end{array}$ & $(\mathrm{T})$ & 8.58 & 7.41 & 6.85 & 6.3 \\
\hline Injection current & (A) & 761.78 & 761.78 & 763.07 & 346.5 \\
\hline Ultimate current & (A) & 12840 & 12840 & 12810 & 5820 \\
\hline$P_{s, h}$ & $(\mathrm{~mm})$ & 23.42 & 23.33 & 23.33 & 13.57 \\
\hline$P_{s, i}$ & $(\mathrm{~mm})$ & 27.2 & 26.53 & 26.53 & 15.42 \\
\hline$P_{i, h}$ & $(\mathrm{~mm})$ & 6.8 & 6.63 & 6.63 & 3.86 \\
\hline$P_{i, b}$ (conservative, see IV.B) & $(\mathrm{mm})$ & 2.6 & 0.44 & 0.43 & 0 \\
\hline
\end{tabular}

the impact of the He phase transition is limited between these time scales, $400 \mu \mathrm{s}$ and $5 \mathrm{~s}$.

The value of the enthalpy of the dry cable gives the lower limit of the stability margin. It can be demonstrated that the actual value of the stability margin is always higher than this limit, even for very short pulse. This is due to the time constant associated with the "decision time", i.e. the time the system waits before "deciding" to quench or not. The difference between these values can be very small as in the case of high current regime because the "decision time" becomes very short. On the contrary the value of the enthalpy of the wet cable is not an upper limit for the system. The total enthalpy of the solid and liquid can be exceeded for long enough pulses. This is due to the presence of an external reservoir, the super-fluid He bath outside the insulation. In fact in case this term and the boiling phases are suppressed, as presented in the third curve of Fig. 2, the value of the stability margin cannot exceed the enthalpy of the wet cable.

Fig. 3 shows the stability margin calculated for 11850 A together with its associated quench power (minimum energy causing a quench divided by the heating time): for long heating times the power needed to quench the cable decreases and reaches asymptotically the steady state value. This calculated quench power is in agreement with steady state measurements performed in similar conditions [11].

From the above considerations it is possible to understand the different behavior observed at low current regimes $(<35 \%$ of the critical current). Since the "decision time" is noticeably longer, the stability margin is much higher than at high currents, and it is independent on the heating time until this last one does not exceed the "decision time". This reduces the impact of the heat transfer model for such heating times.

\section{B. Impact of the Cooling Surface}

The influence of the He micro-channels located between the cables in the coil and of the He channels network through the cable insulation have been preliminary investigated. In fact, their actual impact is unknown, so that we finally ignore the

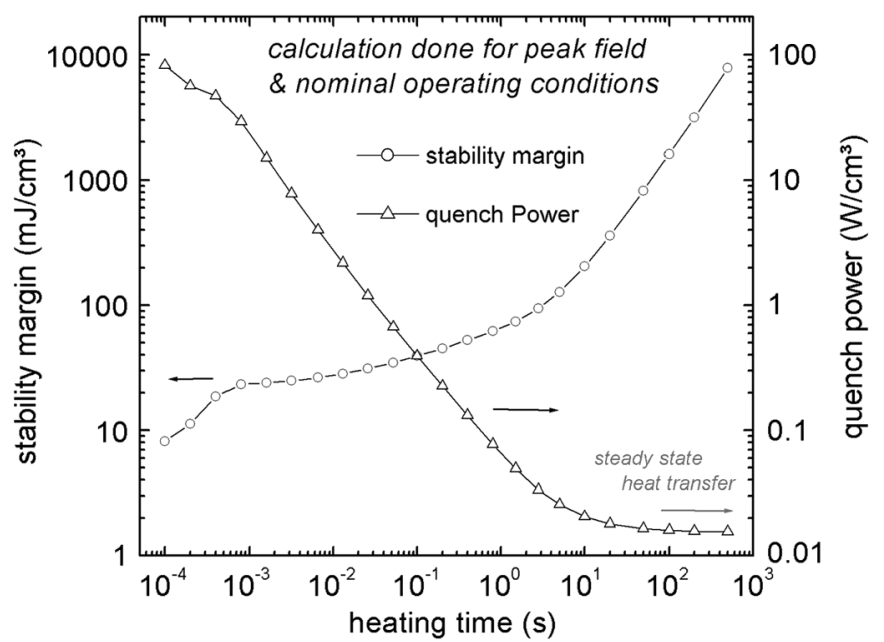

Fig. 3. The stability margin and the quench power of cable 1 are estimated for different heating times. The power decreases for higher heating time and gets flat, while it is reaching asymptotically the steady state heat transfer regime.

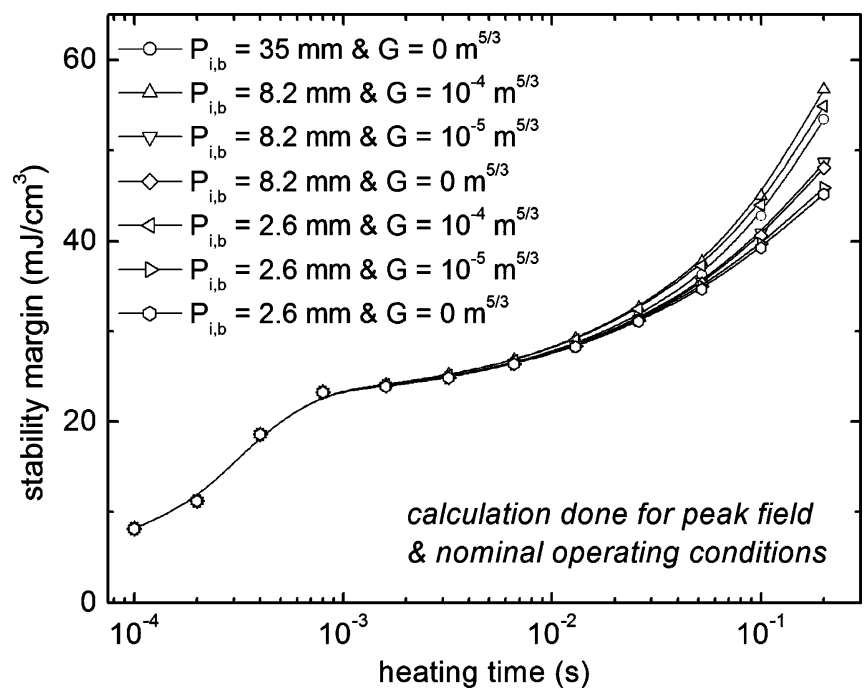

Fig. 4. Stability margin of cable 1 at nominal current and peak field as a function of heating time. Different $p_{i, b}$ and sizes of the He channels network through the insulation have been considered.

effectiveness of these two heat transfer mechanisms. The parametric study presented in Fig. 4 points out the importance of the actual $p_{i, b}$. The unrealistic value of $35 \mathrm{~mm}$, corresponding to an entirely wetted insulation, allows a better stability than in the case of not-effective $\mu$-channels $\left(p_{i, b}=2.6 \mathrm{~mm}\right)$. The case of effective micro-channels $\left(p_{i, b}=8.2 \mathrm{~mm}\right)$ is in between. It is clear that this influence becomes very important for long perturbation times. On the other hand, the actual size of the He channels network (represented by $G$ ) starts playing an appreciable role for values above $10^{-5} \mathrm{~m}^{5 / 3}$.

In the following calculations a conservative case has been considered, where $\left(p_{i, b}=2.6 \mathrm{~mm}\right)$ and $G=0 \mathrm{~m}^{5 / 3}$.

\section{Systematic Calculation of the Stability Margins}

All the cables presented in Table I have been considered for the systematic calculation of the stability margin of the LHC cables. Besides cable 1, the outer layer cable of the main dipoles (cable 2), the cable of the main quadrupoles (cable 3 ) and the 


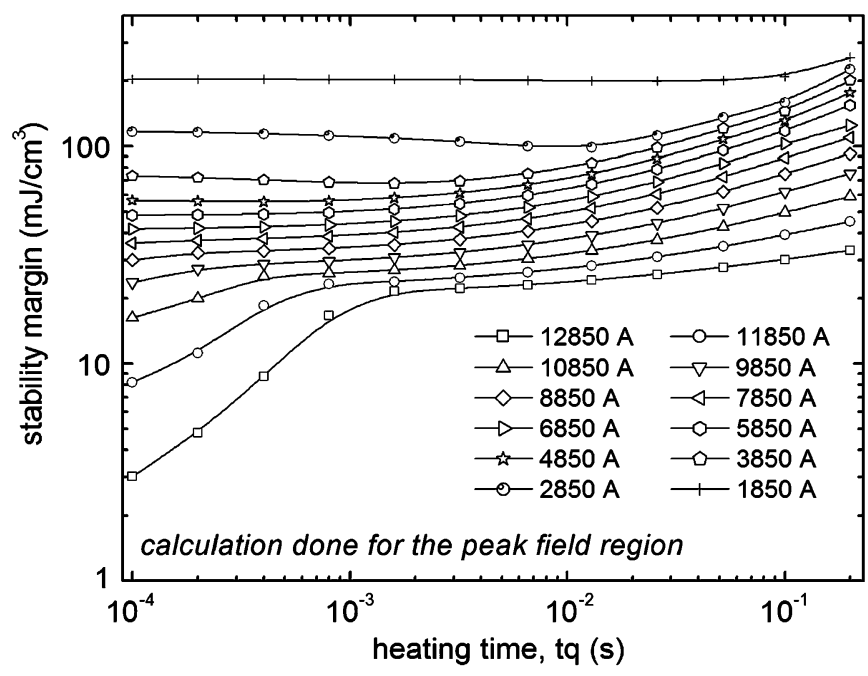

Fig. 5. Stability margin of cable 1 for the peak field region of the MB magnet as a function of heating time and for different current levels.

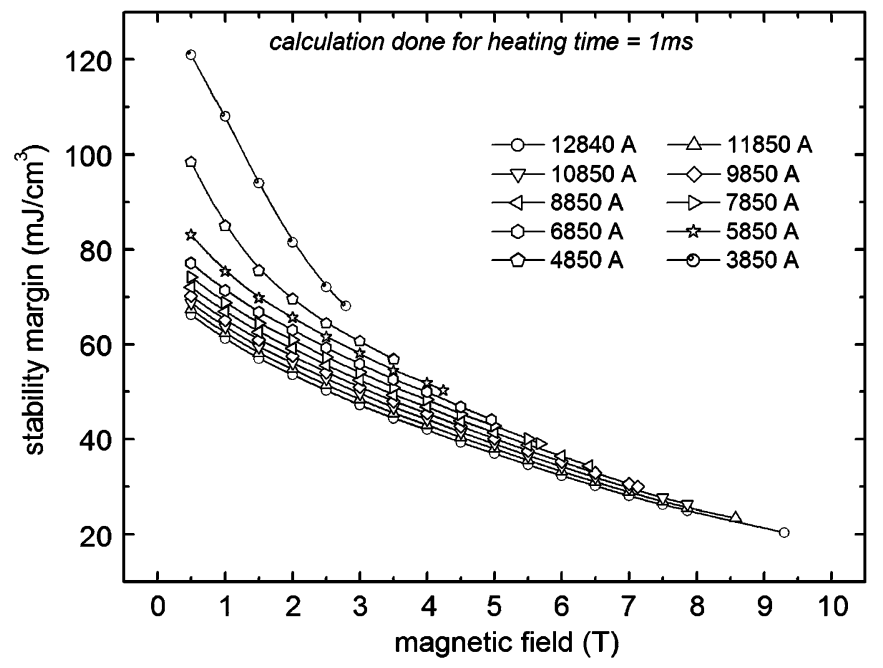

Fig. 6. The stability margin of cable 1 is presented as a function of magnetic field and for several current levels. The magnetic field ranges between $0.5 \mathrm{~T}$ and the peak field for a given current.

cable of the insertion quadrupoles MQM (cable 4) are also considered. The results for cable 1 are presented in Fig. 5. For short heating time the sensitivity of the stability margin to the pulse duration is relevant only for high current regime, while for lower current values the effect becomes smaller, as previously explained. The well pronounced knee at $12850 \mathrm{~A}$ (around $1 \mathrm{~ms}$ ) shifts at lower currents and becomes less relevant while current decreases and almost disappears below $8 \mathrm{kA}$. The local minimum observed for low currents is due to the actual model used for the heat transfer coefficient in boundary layer. This can be considered as a possible artifact of such simplified approach while the more general one mitigates this effect and its implementation is in progress.

The stability margin for a given cable has been estimated as a function of the heating time, current and magnetic field. An example is presented in Fig. 6 for cable 1 for a heating time of $1 \mathrm{~ms}$. With such information it is easy to interpolate the results at any field for any cable in the magnet cross section. Indeed, even if the most critical cable is the one at the peak magnetic

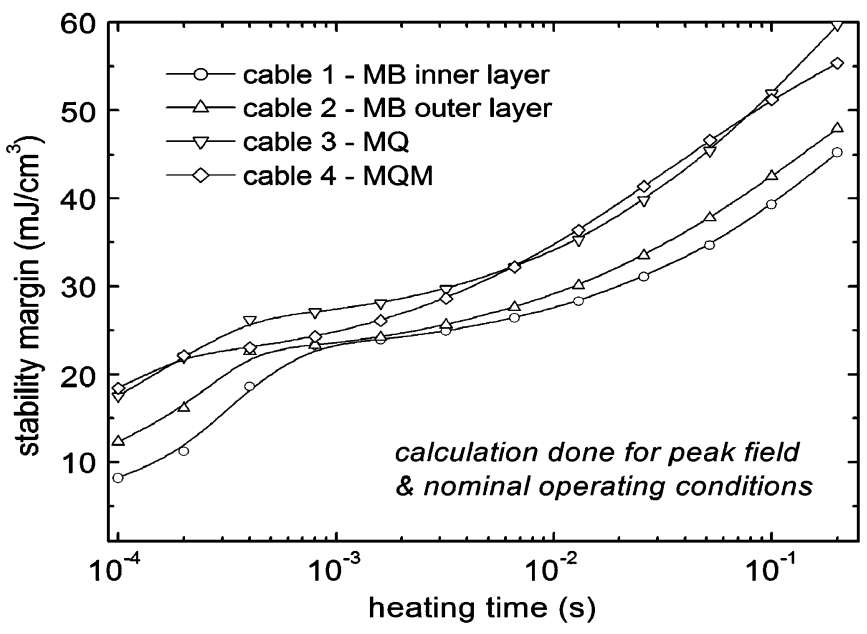

Fig. 7. Stability margin of all the cables presented in Table I at their nominal current and peak field as a function of heating time.

field located in the upper pole, other region of the magnet like for instance the mid-plane cables of the dipoles can be probably more affected by beam loss heat deposition.

Fig. 7 presents the stability margin of the above mentioned cables at their nominal operating conditions as a function of the heating time. It shows a better stability of the quadrupole cables compared to the dipole ones.

Calculations on the LHC SC cables working at $4.5 \mathrm{~K}$ are ongoing and will be soon available, together with the detailed calculations relative to all cables working at $1.9 \mathrm{~K}$ in all the possible operating conditions.

\section{CONCLUSION}

A 0-D thermal model has been built to study the stability of the LHC SC magnets, taking into account all relevant He phases discussed in the literature. The concept of "decision time" has been introduced to explain the behavior of a cable against transient disturbances. Further research is going on.

A complete scan of the stability margins of the LHC cables working at $1.9 \mathrm{~K}$ has been obtained, with respect to several parameters. It will allow setting the BLM system, thus being crucial for the correct exploitation of the LHC.

Improvements of the heat transfer model are ongoing, together with the increase of the complexity of the 0-D approach. Indeed, the great number of simulations needed to get a comprehensive picture of the stability of the LHC cables requires a numerical model of low computational cost, but a more accurate multi-strand approach with periodical boundary conditions would better describe the internal structure of the cable and the predicted shape of a beam loss energy deposit.

Stability measurements for steady state energy deposition have been performed on few LHC magnets. Means to experimentally validate the consequences of transient energy deposition are under study.

\section{ACKNOWLEDGMENT}

The authors thank D. Leroy, R. Schmidt, L. Walckiers, A. Ballarino, S. March, M. Pojer, M. Di Castro, T. Boutboul, and P. Pugnat from CERN for useful suggestions. 


\section{REFERENCES}

[1] The LHC Design Report CERN-2004-003, vol. 1, The LHC main ring.

[2] B. Dehning et al., Design of the Beam Loss Monitoring System for the LHC Ring LHC Project report 781.

[3] A. Fasso et al., "The FLUKA code: Present application and future developments," in CHEP03, La Jolla, CA, USA, March 24-28, 2003.

[4] P. Bauer et al., "Minimum quench energies of LHC strands," IEEE Trans. Appl. Supercond., vol. 9, no. 2, pp. 1137-1140, 1999.

[5] D. E. Baynham et al., "Transient stability of LHC strands," IEEE Trans. Appl. Supercond., vol. 9, no. 2, pp. 1109-1112, 1999.

[6] M. N. Wilson and R. Wolf, "Calculation of minimum quench energies in Rutherford cables," IEEE Trans. Appl. Sup., vol. 7, no. 2, pp. 950-953, 1997.

[7] L. Bottura, M. Calvi, and A. Siemko, "Stability analysis of the LHC cables," Cryogenics, vol. 46, pp. 481-493, 2006.

[8] M. Breschi, P. P. Granieri, M. Calvi, M. Coccoli, and L. Bottura, "Quench propagation and stability analysis of Rutherford resistive core cables," Cryogenics, vol. 46, pp. 606-614, 2006.

[9] ZERODEE Software. CryoSoft, France, 2001.
[10] B. Baudouy et al., "He II heat transfer through superconducting cables electrical insulation," Cryogenics, vol. 40, pp. 127-136, 2000.

[11] D. Bocian et al., "Modelling of quench limit for steady state heat deposits in LHC magnets," IEEE Trans. Appl. Sup., to be published.

[12] V. D. Arp, "Stability and thermal quenches in forced-cooled superconducting cables," in Proceeding of 1980 Superconducting MHD Magnet Design Conference, Cambridge, MA, 1980, pp. 142-157, MIT.

[13] C. Schmidt, Review of Steady State and Transient Heat Transfer in Pool Boiling He I. Saclay, France: International Institute of Refrigeration: Commision A1/2-Saclay, 1981, pp. 17-31.

[14] M. Breschi et al., "Minimum quench energy and early quench development in NbTi superconducting strands," IEEE Trans. Appl. Sup., vol. 17, no. 2, pp. 2702-2705, 2007.

[15] M. Nishi et al., "Boiling heat transfer characteristics in narrow cooling channel," IEEE Trans. Magn., vol. 19, no. 3, pp. 390-393, 1983.

[16] Y. Iwasa and B. A. Apgar, "Transient heat transfer to liquid He from bare copper surfaces to liquid $\mathrm{He}$ in a vertical orientation-I: Film boiling regime," Cryogenics, vol. 18, pp. 267-275, 1978.

[17] Z. Chen and S. W. Van Sciver, "Channel heat transfer in He II—Steady state orientation dependence," Cryogenics, vol. 27, pp. 635-640, 1987. 